\title{
Exploring the Educational Value of Mathematical Beauty and Effective Ways of Discovering Mathematical Beauty
}

\author{
Cunrong Wang* \\ School of Mathematics and Statistics, Qilu University of Technology (Shandong Academy of Sciences), Jinan 250353, China \\ *Corresponding author: Cunrong Wang, 1191984686@qq.com

\begin{abstract}
The precision of mathematical reasoning, the abstractness of mathematical language, the profundity of mathematical thought and method, as well as the excessive formalization of mathematics teaching have formed an impassable gap, hindering students in approaching mathematics. This has concealed the beauty of mathematics and the light of mathematical culture. However, if students are able to cross this gap, they would find that mathematics is a vast world full of vitality, imagination, wisdom, poetry, and beauty. The pursuit of mathematical beauty is one of the motivations for scientists to research this field. Experiencing mathematical beauty is of great significance to students' learning and growth. In teaching, the value of mathematical beauty is explored, such as stimulating emotions, opening up to the truth, and cultivating goodness. Several effective ways are suggested in this article to guide students to discover the mathematical beauty in life while finding it in problem-solving methods and exploring it in knowledge systems.
\end{abstract}

Keywords: Mathematical beauty; Educational value; Effective way

Publication date: October 2021; Online publication: October 29, 2021

\section{Introduction}

The precision of mathematical reasoning, the abstractness of mathematical language, the profundity of mathematical thought and method, as well as the excessive formalization of mathematics teaching have formed an impassable gap, hindering students in approaching mathematics. This has concealed the beauty of mathematics and the light of mathematical culture. Many people lack deep appreciation of the beauty of mathematics.

However, if students are able to cross this gap, they would find that mathematics is a vast world full of vitality, imagination, wisdom, poetry, and beauty. The pursuit of mathematical beauty is one of the motivations for scientists to research the mathematical field. Experiencing mathematical beauty is of great significance to students' learning and growth. In teaching, the value of mathematical beauty is explored while paving an effective way to discover mathematical beauty.

\section{Educational value of mathematical beauty}

Mathematics is a formalized reasoning, not an empirical generalization ${ }^{[1]}$. Although mathematics is a "rational" science, it is still a science of beauty, unity, simplicity, symmetry, and singularity. The English mathematician and philosopher, Bertrand Russell, once said, "Mathematics, when viewed correctly, is of supreme beauty [2]." The German mathematician, Klein, said, "Music stimulates or soothes feelings, painting makes people happy, poetry moves the heart, philosophy provides wisdom, science improves material life, but mathematics gives all of the above ${ }^{[3]}$." Although Klein did not directly state the role and 
significance of mathematical beauty, it leaves a deep inspiration. The value of mathematical beauty can be explored from three aspects.

\subsection{Stimulating emotions with mathematical beauty}

The beauty of mathematics arouses the intense pleasure of mathematicians' intelligence which gives them the indomitable perseverance of mathematical creation, the courage to overcome difficulties, and the strength to climb the scientific peak. This is similar to what Poincare has mentioned: "People devote themselves to the study of mathematics, which is actually the search for the beauty of mathematics and the harmonious meaning of the universe ${ }^{[4]}$."

In mathematics teaching, the "affection" in the passion of aesthetics does not only refer to the students' feelings toward mathematics, but also to the feelings between teachers and students. First, through the understanding of mathematical beauty, its true meaning can be realized, and the appreciation as well as pursuit of beauty can be stimulated. Students would be able to experience the superiority of numbers created by the heavens and earth, the profoundness of mathematics created by mathematicians, and the joy of learning mathematics. In this way, students would be attracted to mathematics, develop a fondness toward it, and enjoy it while learning mathematics. Second, the teaching methods that teachers use to guide students to discover and appreciate mathematical beauty create a relaxed, harmonious, and cheerful environment. They not only create an equal and democratic relationship between teachers and students, but also a harmonious and cooperative relationship among students.

\subsection{Discovering the truth with mathematical beauty}

"Enlightening truth with beauty" refers to discovering mathematical truth with beautiful thoughts as well as discovering mathematical laws and solving mathematical problems with beautiful methods. Secondly, it refers to the beauty of mathematical methods of thought. In regard to that, shortcuts can be explored to solve problems by using simple beauty, inspire problem-solving ideas by using harmony beauty, simplify the problem-solving process by using symmetry beauty, summarize problem-solving rules by using similarity beauty, and find solving loopholes by using rigorous beauty ${ }^{[5]}$. For example, the establishment of projective geometry can be regarded as a branch of mathematics formed by the pursuit of symmetry beauty in a certain sense. In contrast to Euclidean geometry, an important feature of projective geometry developed by the French mathematician, De Sager, who introduced the point at infinity, is the symmetry of points and lines ${ }^{[6]}$. Non-Euclidean geometry, thus, can be seen as a result of the pursuit of simple beauty by our mathematical ancestors.

\subsection{Cultivating goodness with mathematical beauty}

In fact, the beauty of mathematics not only lies in itself. Fundamentally, the beauty of mathematics represents the spiritual power of the human nature. People who strive for progress in mathematics all their lives add luster to the development of the discipline with their scientific spirit and indomitable perseverance. The ancient Greek mathematician, Proclus, proclaimed, "Wherever there is number, there is beauty."

Generations of scientists have spent more than 2,000 years exploring the proof of the fifth postulate in geometry. Lobachevsky, who dared to challenge the Euclidean geometry, countered Kant's philosophy and was willing to struggle against adversity all his life. He was considered the founder of non-Euclidean geometry. Cantor, who was still full of confidence and stood firm in the face of criticism and accusations, was the founder of set theory. Whether in the heat of summer or the coldness of winter, the Chinese mathematician, Jingrun Chen, who immersed himself in his studies, finally cracked the Goldbach conjecture. 
Their passion and dedication to mathematical research do not only help us to understand and surpass ourselves, but also establish the correct values and outlook on life and the world.

\section{Effective ways in discovering mathematical beauty}

Chong Wang, a famous scholar in ancient China, once said, "Those who wade in shallow water will see shrimps, but those who go deep enough will see fishes and soft-shelled turtles, and even river dragons." The level of experiencing mathematical beauty depends on an individual's understanding of the mathematical knowledge system, emotional response to mathematics, mathematical accomplishments, as well as aesthetic interests and abilities. Mathematics teachers should place emphasis on their leading role in guiding students to discover the mathematical beauty of life, to seek it in arithmetic and through reasoning, as well as to embed it in the mathematical knowledge system.

\subsection{Discovering the mathematical beauty of life}

Mathematical beauty is not purely subjective and unpredictable. Mathematical beauty has its definite, objective content. It comes from the beauty of nature, which is closely related to the beauty in our daily lives.

This can be exemplified in the secrets of the honeycomb. The structure of the honeycomb is a hexagonal column, which does not only radiate regularity and symmetry, but also make the most efficient use of materials. It can be confirmed that the structure of the honeycomb completely conforms to the optimization theory by calculating its surface area.

Another example is the wonder of the number " 0.618 " or the golden ratio. Pythagoras once called the golden ratio the most exquisite proportion in the world. The number 0.618 is wonderful as it can be found everywhere, as far as the ancient Egyptian pyramids, the Parthenon in ancient Greece, the Taj Mahal in India, the Eiffel Tower in Paris, the goddess of wisdom, Athena, the sun god, Apollo, and in every leaf around us. This ratio is implicit everywhere ${ }^{[7]}$.

The beauty of curves and surfaces provides another example. Graceful curves also bring people great joy. It is derived from the four-leaved rose in nature and applied in architecture in the form of logarithmic spirals and artificial super-elliptic curves. There is also the famous Möbius strip. The three-leaf kink of the China Science and Technology Museum is derived from the Möbius strip. Although it is a threedimensional figure, there is only one side; that is, a single side, which indicates that science is borderless. There is no boundary between various sciences; they are continuous with each other. Science and art are also linked to one another ${ }^{[8]}$. There is a peculiar mathematical monument featuring an eight-foot-high stainless steel Möbius circle which revolves slowly day and night at the entrance to a museum in Washington. This feature does not only provide aesthetic enjoyment, but its perpetual motion also signifies the perpetual motion of humankind.

There are countless examples of mathematical beauty in life, and it is possible to appreciate them if we look hard enough. It is necessary to spread the word about the beauty of mathematics whenever they are appreciated or enjoyed. Mathematics should be made a topic of delightful after-dinner conversation just as we do for poetry, music, and art.

\subsection{Looking for mathematical beauty in problem-solving}

Diderot once pointed out, "A beautiful problem in mathematics is a difficult problem to solve. A beautiful solution is a simple answer to a difficult, complex problem ${ }^{[9]}$." Problems are at the heart of mathematics, and problem-solving is its main melody. The ideological method of problem-solving is the essence of mathematics. The sophistication of algorithmic mathematics and the philosophy of speculative mathematics 
can be appreciated through several examples.

First, ancient Chinese mathematics emphasizes more on calculation. Their calculation methods and ideas highlight the peculiar beauty of mathematics. Their answers reveal the dialectical thinking of ancient Chinese mathematicians.

One example is the widely discussed problem about "chickens and rabbits in a cage" in Sun Tzu Suan Jing. Both chickens and rabbits are cooped up together; there are thirty-five heads at the top and ninetyfour feet at the bottom. How many chickens and rabbits are there? The answer to this problem is 23 chickens and 12 rabbits.

As there was no algebraic method available then, people had to solve this problem arithmetically. Sun Tzu's Suanjing provided a solution to this problem: "The number of feet is first divided into two to obtain forty-seven; the number of rabbits is obtained by subtracting thirty-five from forty-seven; the number of rabbits from the head count of thirty-five is subtracted to obtain the number of chickens." In this solution, every chicken stands on one foot, and every rabbit worships the moon. The number of heads and feet per chicken is equal, and the number of feet per rabbit is twice as many as the number of heads.

This kind of "half-foot art" embodies the thought of opposition and unity, which seeks the mutual transformation of the two in contradiction. Therefore, this problem-solving method can be said to be the product of dialectical thinking ${ }^{[10]}$.

Second, speculative mathematics yield to the speculative beauty of mathematical thought. Wonderful solutions shine with the light of wisdom and often endow people with spiritual pleasure and satisfaction.

For example, suppose there is a glass of white wine and the same amount of white wine. A spoonful of white wine is added to the red wine. The same amount of the mixture is then added to the white wine. Is there more or less red wine in the white-wine glass than there is white wine in the red-wine glass ${ }^{[11]}$ ?

Looking at this problem, calculation is necessary and at the same time, several conditions should be proposed. How much is a glass of wine? Is there a good mix? The conditions that need to be defined are not provided in the problem. Hence, it is impossible to solve this problem by mere computation.

It is only possible to apply speculative methods. Imagine that the white wine and red wine in each glass are separated. The red wine in the white-wine glass is the missing part of the red wine in the red-wine glass, which is now replaced by the white wine. Thus, there is as much red wine in the white-wine glass as there is white wine in the red-wine glass.

\subsection{Exploring mathematical beauty in knowledge systems}

The knowledge of definitions, theorems, laws, and formulae in mathematics obscures and dispels the charm of mathematics. However, mathematical knowledge, which can often seem dry and boring, conveys the coldness and seriousness of mathematical beauty.

There is no mathematical beauty without mathematical knowledge. In order to explore the beauty in the mathematical knowledge system, it is not only important to combine students' existing mathematical knowledge with their experiences, but also the mathematics topics which they are learning. In fact, the mathematics curriculum is full of simplicity, symmetry, and harmony. In aesthetic education, the understanding of the harmonious characteristics of aesthetic objects would help students develop harmoniously and grow in a healthy way ${ }^{[12]}$. The unified beauty of mathematics is examined in a detailed manner.

Hegel stated that harmony, on the one hand, reveals the totality of the essential differences, while, on the other hand, it eliminates the pure opposition of those differences ${ }^{[13]}$. Unifying beauty in mathematics refers to the internal connection or common law between content and form as well as between a part and a whole. 
For example, the mystery of the unit circle ${ }^{[14]}$. While straight lines offer fortitude, curves are gentle. Axial symmetry and central symmetry provide visual pleasure. The Pythagoras school of ancient Greece thought has proposed that the most beautiful of all three-dimensional figures is the sphere, while the most beautiful of all flat shapes is the circle. A unit circle is a circle centered at the origin with a radius of 1 unit length. The unit circle is not only beautiful, but also contains infinite secrets.

The trigonometric function defined by the directed line segment in the unit circle is intuitive. The image of the trigonometric function created by translation of the trigonometric correspondence line resembles a folded magic picture scroll. Sine and cosine curves are like rolling waves. Tangent and cotangent curves represent beautiful music being played out. The length of the decagon inside the unit circle is the golden number. Therefore, it is said that the unit circle contains infinite secrets.

Another example is the basic formula of calculus. First, the focus is on the origin of the definite integral. Finding the area of a curved trapezoid, the distance traveled by an object moving at variable speed, and the work done by a variable force are different problems. These unrelated problems surprisingly embody consistent quantitative laws because they all boil down to dividing, approximating, summing, and taking the limit of a certain quantity in the problem. This problem-solving model is so widely used that the predecessors of calculus abstracted the definition of the definite integral.

Therefore, there is a profound realization that mathematics reflects objective reality from the aspect of quantity; that is to say, in mathematical abstractions, only quantitative properties are retained whereas qualitative properties are discarded altogether ${ }^{[15]}$. The regularity of mathematics should be appreciated ${ }^{[16]}$. Mathematics is the science of patterns, and patterns that reflect a wonderful and colorful world are the soul of mathematical thought.

Secondly, the definition of the definite integral has been examined. The definite integral and primitive functions are not directly related. However, the basic formula of calculus perfectly connects the irrelevant definite integral with the original function, which does not only simplify the calculation of the definite integral, but also lay a solid foundation for the application of calculus. From now on, the universality of the mathematical method of thought can be appreciated and the unified beauty of mathematics can be witnessed.

\section{Conclusion}

Mathematics is beautiful. If we really appreciate the beauty of mathematics, curves are not only soft and smooth shapes, but they also provide rich and profound meanings. Mathematical symbols are no longer dull and considered as a difficult language, but they are rational notes that constitute the symphony of nature. Mathematics is no longer a cold and ugly monster, but a graceful and charming beauty. Mathematical beauty is all around us, let us enjoy the beauty of mathematics.

\section{Funding}

This research was supported by the 2020 Qilu University of Technology (Shandong Academy of Sciences) Teaching Research Project - Theoretical and Practical Research of Higher Mathematics Curriculum Ideological Politics (2020szzx23).

\section{Disclosure statement}

The author declares that there is no conflict of interest.

\section{References}

[1] Chen H, Zhang X, 2008, On the Essential Attribute of Mathematical Beauty. Journal of Mathematics 
Education, (5): 28-30.

[2] Deng D, 1990, Mathematics and Culture, Peking University press, Beijing, 41.

[3] Several Types of Mathematical Beauty. https://www.docin.com/p-21423184.html (accessed on May 27, 2019).

[4] Middle School Mathematics Room, 2003, Mathematical Culture, People's Education Press, Beijing, 179.

[5] Ni B, 2010, The Application of Mathematical Beauty in Solving Mathematical Problems in High School. Education Theory and Practice, (8): 50-51.

[6] Zheng Y, 2006, Introduction to Mathematical Methods, Zhejiang Education Press, Hangzhou, 58.

[7] The Golden Ratio. https://www.docin.com/p-8237915.html (accessed on March 19, 2015).

[8] Three Blades Kink. https://baike.baidu.com/item (accessed on November 7, 2011).

[9] Zheng Y, 2006, Introduction to Mathematical Methods, Zhejiang Education Press, Hangzhou, 61.

[10] Gao S, 2019, Dialectical Thinking in the Problem of Chicken and Rabbit Cooped Up - Course Teaching Material. Teaching Method, (9): 88-93.

[11] Zhang L, Tang R, 1996, Mathematics Education, Jiangxi Education Press, Nanchang, 181-182.

[12] Gu M, 1997, The Comprehensive Dictionary of Education, Shanghai Education Press, Shanghai, 562.

[13] Hegel C, 1979, Aesthetics (Volume 1), The Commercial Press, Beijing, 176.

[14] Yi N, 2004, Mathematical Beauty, Science Press, Beijing, 220-221.

[15] Zheng Y, 2005, Mathematical Culturology, Sichuan Education Press, Chengdu, 29-30.

[16] Zhang X, Cheng M, 1994, Beauty theory of Physical Science, Shaanxi People's Publishing House, Xi'an, 17. 